\title{
Antiviral activity of Carica papaya extract against experimental dengue virus 1 infection
}

\author{
MO Galappaththi, WMA Indeewari, AMSB Abeykoon, MGCM Muthuwaththa, VK Kothwela, \\ AHMLN Kumari, HDRPD Harasgama, F Noordeen
}

\section{Introduction}

Dengue is a major public health problem in tropical countries including Sri Lanka. There are no specific antiviral drugs or an effective vaccine available against dengue as yet. Plant derived compounds are an important option for development of new drugs. Carica papaya of the family Caricaceae is a traditionally used medicinal plant to treat dengue. The objective of this research was to determine the antiviral activity of $C$. papaya leaf extracts against dengue virus-1 (DENV1). As a first step of exploring the antiviral activity, the cytopathic effects of the $C$. papaya leaf extract was done to select a minimum toxic concentration against the experimental DENV-1 infection in $\mathrm{C} 6 / 36$ cells.

\section{Methods}

First, $C$. papaya leaf extract was prepared in two-fold dilution series from neat to 1/1024. Then, two 24-well cell culture plates containing C6/36 cells infected with DENV-1 were treated with C.papaya leaf extracts at different concentrations. The cells were then harvested with the supernatant for testing. Viral RNA extracted (QIAGEN, Germany) from DENV-1 infected untreated control and four infected treated samples with $C$. papaya leaf extracts were subjected to the qRT-PCR. The analytical system based negative control, positive control and four standard dilutions of positive control in replicates were used for quantifying the DENV-1 RNA using the Dengue generic real time RT-PCR (Liferiver, China) with an analytical sensitivity of $1 \times 10^{3}$ copies/ mL.

\section{Results}

According to cell morphology, less damage was observed in 1/16 - 1/512 C. papaya leaf extract dilutions. Thus 1/32- 1/256 dilutions were selected for treating the experimental DENV-1 infection. qRT-PCR showed DENV RNA in all 4 standards controls (SD1-10 ${ }^{7}, \mathrm{SD}_{2}-10^{6}, \mathrm{SD} 3-10^{5}$,

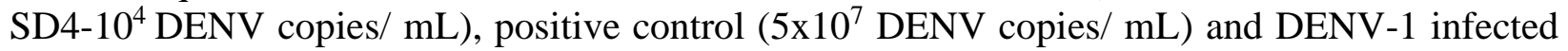
untreated control ( $3 \times 10^{4}$ copies/ mL). qRT-PCR did not show DENV RNA in negative control and four DENV-1 treated samples with 4 different concentrations of $C$. papaya leaf extracts.

\section{Conclusions}

According to qRT-PCR results, DENV-1 infection is inhibited by treatment with $C$. papaya extract in all four concentrations tested in the current experiment. This data suggests the potential of $C$. papaya leaf extract for future development of anti-viral drugs against DENV-1. Experiments are in progress to test $C$. papaya leaf extract against DENV-2, DENV-3 and DENV-4.

Keywords: Antiviral activity, C. papayaleaf extract, DENV-1 infection, qRT-PCR

Funding: University of Peradeniya (Grant No URG/2016/47/M)

Department of Microbiology, Faculty of Medicine, University of Peradeniya, Peradeniya, Sri Lanka Address for correspondence: Prof Faseeha Noordeen. Telephone: +94772293301

Email: faseeha.noordeen12@gmail.com iD https://orcid.org/0000-0002-2018-0606 